\title{
Progress on Research for the Treatment of HIV/AIDS with Traditional Chinese Medicine in China
}

\author{
Jian Wang, Bi Yan Liang \\ Traditional Chinese Medicine Center for AIDS Prevention and Treatment, China Academy of Chinese Medicine Sciences, Beijing \\ China. \\ Email: 62tiger@163.com
}

Received June 28 ${ }^{\text {th }}, 2011$; revised August $1^{\text {st }}, 2011$; accepted August $8^{\text {th }}, 2011$.

\begin{abstract}
The mortality rate associated with HIV/AIDS has decreased dramatically after highly active antiretroviral therapy (HAART). However, issues such as viral reservoirs, drug resistance and side effects have led to a significant crisis in the management of HIV/AIDS. It has become evident that HAART does not offer a complete solution to the problem. Therefore, additional and alternative therapeutic strategies urgently need to be explored. Traditional Chinese medicine (TCM) is one of the mainstays of complementary and alternative medicine, and its use has aroused increasing attention. This paper reviews the recent progress in the use of TCM, from single herbs, herbal ingredients, compound Chinese herbal medicine, acupuncture and medical care, for the treatment of HIV/AIDS in China. We also review evaluation systems that assess the efficacy of TCM.
\end{abstract}

Keywords: HIV/AIDS, Traditional Chinese Medicine (TCM)

\section{Introduction}

Acquired immunodeficiency syndrome (AIDS) is a set of symptoms and infections that are life threatening and result from damage to the human immune system. It is caused by the human immunodeficiency virus (HIV), and was first identified in 1981. It is the fourth biggest killer worldwide and the most quickly spreading disease of the century [1,2]. It is now 26 years since the first case of AIDS was reported in 1985 in China. According to recent reports from the Ministry of Health in China [3], by the end of October 2010, the cumulative total of reported HIV-positive cases was 370,393, including 132,440 AIDS cases and 68,315 recorded deaths. The AIDS epidemic in China is rising, and prevention and treatment are major concerns.

Highly active antiretroviral therapy (HAART) is very effective in suppressing viral replication and has led to a significant reduction in the mortality rate of the disease, an increase in the life expectancy of HIV/AIDS patients, and an improvement in quality of life (QoL) of these patients [4-6]. However, issues such as, viral reservoirs, drug resistance, high dosage and drug costs, have led to a significant crisis in the management of HIV/AIDS, particularly in developing nations, where there is the greatest need [7-9]. It has become evident that HAART does not offer a complete solution to the problem. Therefore, additional and alternative anti-HIV therapeutic strategies urgently need to be explored [10]. Traditional Chinese medicine (TCM) is one of the mainstays of complementary and alternative medicine, and its use has increased [11]. Generally, people with HIV/AIDS use TCM for four main reasons: to enhance their immune function, to treat symptoms, to improve their QoL, and to reduce side effects related to medications $[12,13]$. The objective of this article is to review the recent use of TCM in HIV/ AIDS in China.

According to the review by Wang Jian [14], research on TCM for the treatment of HIV/AIDS has gone through three stages: a perceptual stage, a preliminary study stage, and the stage of gradually standardizing and improving treatment.

\section{Chinese Herbal Medicine}

Treatment of HIV/AIDS remains inadequate because the HIV virus mutates rapidly, appropriate animal models 
are lacking, development of a vaccine has great difficulties and existing anti-viral treatments cannot fundamentally destroy the virus. There may be, therefore, a potential role of TCM in the treatment of HIV/AIDS. After years of clinical applications, it should be possible to develop effective drugs from the numerous existing natural traditional medicines for the treatment of HIV/ AIDS. On this basis, research on natural medicines that suppress HIV/AIDS is currently being carried out all over the world. In particular, Professor Lv, Professor Wang and colleagues of the China Academy of Chinese Medicine Sciences, Professor Luo of Kunming Institute of Botany, and other scholars have been performing research in this area for the past 20 years [15].

There are three aspects to the research on TCM for the treatment of HIV/AIDS: firstly, single herbs are screened; secondly, the active herbal ingredients are studied, and thirdly, compounds of Chinese herbal medicine are studied.

\subsection{Research on Single Herbs}

In the past 20 years, many scholars who conducted research on Chinese herbal medicines found that a variety of traditional medicine to some extent had inhibitory effects on HIV and improved immune function. Thousands of herbs have been screened for anti-HIV activity. Over 140 kinds of herbs have been shown to have inhibitory effects on HIV. Of those herbs, more than 20 species showed strong anti-HIV activity [15,16] (Table
1). Rebuilding the immune system is crucial because AIDS patients have severely impaired immune function. Almost all tonics used in TCM had varying degrees of effectiveness in enhancing immune function $[17,18]$.

\subsection{Research on Herbal Ingredients (Table 2)}

At present effective extracts of herbal compounds include 15 polysaccharides, seven alkaloids, 29 flavonoids, 29 terpenes, eight coumarins, eight tannic acids, four plant hemagglutinins, four quinolones, two peptides, and various others [19-21]. The mechanisms of action include the competitive and non-competitive inhibition of reverse transcriptases, proteases, and interference with the viral entry into cells. These promising results support the search for further Chinese herbal medicines that are effective anti-HIV agents.

\subsection{Clinical Studies of Compound Chinese Herbal Medicine}

The first patent approved by the State Food and Drug Administration (SFDA) was for Tangcao tablets, which are used to alleviate the signs and symptoms of HIV/ AIDS. Eleven compounds have obtained clinical approval (Aiqikang granule, Keaite granule, Fufangsanhuangsan, Qiankunning tablets, Aining particles, Aifukang granule, Fufang SH, Quduzengning granule, Aikefuzheng tablets, Aifuping granule, Chuankezhi injection), of which five relatively matured compounds (Qiankunning tablets, Keaite granule, Fufangsanhuangsan, Aining par-

Table 1. Single herbs that have anti-HIV effects.

\begin{tabular}{l}
\hline Single Herbs \\
\hline Licorice, Astragalus, Trichosanthin, Cortex Mori, Lithospermum, Chinese Violet, Polygonum Cuspidate, Scutellaria, Salvia, \\
Bupleurum, Croton, Radix Rhapontici, Prunella Vulgaris, Honeysuckle, Epimedy, Cassia, Dark Plum,Chrysanthenum Anti-HIV activity \\
Indicum, etc.
\end{tabular}

Table 2. Constituent herbal ingredients with anti-HIV activities.

\begin{tabular}{|c|c|c|}
\hline Herbs Ingredients & Herbs & Effect \\
\hline polysaccharides & $\begin{array}{c}\text { Seaweed, Indocalamus, Trihexyphenidyl Grass, Mushrooms, } \\
\text { Prunella Vulgaris, Viola, Eclipta, Aloe, etc. }\end{array}$ & Block HIV virus and cell adsorption \\
\hline protein & Trichosanthes, Bitter Melon, Musk, Pink, etc. & $\begin{array}{l}\text { Inhibition of ribosomal RNA to destroy protein } \\
\text { synthesis of normal chromosome }\end{array}$ \\
\hline alkaloids & Tripterygium, etc. & Anti-HIV activity \\
\hline flavonoids & Snow Lotus, Narrow-Leaf Nettle, etc. & Interfere with the virus and cell integration \\
\hline coumarin & Poon, Musk Root, Narrow Every Celery, Coriander, etc. & HIV reverse transcriptase inhibitors \\
\hline lignans & Cairica, Millettia, Myrobalan, etc. & Anti-HIV-1 replication activity \\
\hline plant haemagglutinin & Skullcap, Turks Rose, Cornflower, Licorice, etc. & Anti-HIV activity \\
\hline terpenes & $\begin{array}{l}\text { Andrographis, Annona, Croton, Almond Shells, Fungus, } \\
\text { Schisandra, Hawthorn, Cynomorium, Horse Chestnut, etc. }\end{array}$ & Multiple targets for HIV replication cycle \\
\hline
\end{tabular}


ticles, Chuankezhi injection) are still in clinical trials.

Two randomized controlled trials (RCTs) have reported that using Chinese herbs (Qiankunning capsules and Compound Sanhuangsan) for 6-7 months significantly reduced viral loads compared with the control group [22,23]. Three RCTs have reported that the use of Chinese herbs (Tangcao tablets, Aining Granule and ZY4) for 6-12 months stabilized and promoted immune function compared with the control group [24-26]. Clinical studies [22-26] have shown that TCM could improve the signs and symptoms of HIV/AIDS (such as fatigue, diarrhea, fever, and rash) and QoL, and have some effect in increasing and stabilizing immune function, but the effect on viral load was not obvious. As the sample sizes of the current clinical studies were small and observation times were short, future studies will need to address these issues. The use of the RCT method of clinical trials would also yield results that are more scientific and objective.

Nine RCTs [27-35] have reported that using Chinese herbs had a positive effect on HIV/AIDS-related diseases, such as oral candidiasis, peripheral neuropathy, pneumonia and diarrhea, and could improve QoL. However, there were some problems with the overall quality of research, which was not high, and study designs were defective (e.g., small number of cases, random applications, short times of observation, blind implementation). Those problems affected the reliability of the conclusions drawn from the studies. Therefore, the results of those studies will need to be further verified in a large sample, and with more rigorous and extensive clinical research. TCM is becoming widely acknowledged abroad for its medicinal benefits. Yang et al. [36] analyzed the dynamic observation results of $\mathrm{CD}^{+}$and $\mathrm{CD}^{+} \mathrm{T}$ lymphocytes from 13 HIV/AIDS patients in Tanzania, who have survived for more than 10 years. The analysis showed that long-term TCM intervention was effective in increasing and maintaining $\mathrm{CD}^{+} \mathrm{T}$ lymphocytes, and activating or adjusting $\mathrm{CD}^{+}$lymphocytes. TCM was also effective in delaying the progression of AIDS, prolonging longevity and had a long-term therapeutic effect on AIDS. Huang et al. [37] analyzed the effectiveness of TCM in the treatment of 729 HIV/AIDS patients in Tanzania. They showed that TCM could improve symptoms and immune function, and the effect was significant in CD4 $<200$ cell $/ \mathrm{mm}^{3}$.

The composition of TCM is complex and diverse, and it is difficult to identify the mechanisms underlying its effects. Thus, convincing scientific data are lacking. Therefore, greater effort is needed for studies on the active ingredients, and in determining their composition, structure, mechanism, and effective dose. In order to achieve the efficacy of western medicines, purified active ingredients are required.

\section{Clinical Studies of Acupuncture}

Clinical data [38] show that acupuncture can enhance the immune system, has anti-viral effects, relieve symptoms of HIV/AIDS and prolong survival time, so that patients can maintain long-term survival despite infected status. Moxibustion treatment also initially showed potential for treating HIV/AIDS. It had features of relieving symptoms quickly without side effects, and was easily performed at low cost.

Four RCTs have reported that moxibustion, with main points Guanyuan (CV4), Shenque (CV8), Zusanli (ST36), Tianshu (ST25), are effective for AIDS-associated diarrhea [39-42].

\section{Medical Care}

In order to implement the State Council "Four Frees and One Care" policy, a pilot project named the "National Free Treating HIV/AIDS with TCM Program" was launched by the State Administration of Traditional Chinese Medicine on August 2004. By the end of October 2010, 14,244 HIV/AIDS patients have been treated with TCM in 19 provinces. Wang et al. [43] collected data from 8946 HIV/AIDS patients treated with TCM, and the results showed that TCM treatment was effective in relieving symptoms such as fever, asthenia, shortness of breath, loss of appetite, diarrhea, and skin rashes, of which the improvement of asthenia was the most significant. The CD4 count in the asymptomatic period which treated with TCM decreased by an average rate of about $12 / \mathrm{mm}^{3} /$ year, less than the average natural rate of 30 $50 / \mathrm{mm}^{3} /$ year[44], whereas that of the AIDS patients which treated with HAART and TCM increased with time. TCM therapy is able to enhance and stabilize the immune function, improve the symptoms and signs, and improve the QoL.

\section{Efficacy Evaluation Standards System}

In order to reflect clinical efficacy of treatment of HIV/ AIDS with TCM scientifically and objectively, evaluation criteria have been revised by the State Administration of Traditional Chinese Medicine in 2007. Using evidence-based medicine methods, they analyzed clinical data obtained from literature and pilot projects carried out since 2004. Expert panels were convened several times. Having collected clinical observations of 512 cases in eight consecutive months in five areas with a high incidence of AIDS (Henan, Yunnan, Guangdong, Anhui and Beijing), "Outcome Assessment System of Treating HIV/AIDS with TCM" was formed. The system evaluated treatment from the perspective of patients, with self-reporting of symptoms, and utilized patient-reported 
outcomes (PRO) measurements, including the WHOQOL-HIV, QoL scale localization studies, clinical staging of disease, and syndrome and evaluation measures. The main difference of evaluation criteria, between the current system and previous systems, was not only confined to laboratory indicators, but also included QoL, survival time and response to treatment. It has been initially approved.

\section{Conclusions}

The unique advantages of treating HIV/AIDS with TCM are that it is people-oriented, holistic, and the treatment is based on syndrome differentiation. It is characterized by low toxicity, persistent effects, enhancement of immune function, improvement of symptoms and QoL and reducing the incidence of opportunistic infections. Although considerable progress has been made in the treatment of HIV/AIDS with TCM, there have been certain problems. Firstly, the experimental studies and clinical applications have focused on developing Chinese medicine compounds, single herbs and effective components, more focusing on anti-HIV functions and the immune system. There has been a lack of the basic theory of TCM, separating from the holistic concepts and treatment based on syndrome differentiation. Secondly, there is a key problem in clarifying the effectiveness of treating HIV/AIDS with TCM. It is not known which aspects and disease stages of HIV/AIDS could be effectively treated with TCM. Furthermore, whether various evaluation indicators can be combined and agreed standards can be established remains to be resolved, and it remains difficult to determine the actual role of TCM using the existing immune parameters. Thirdly, there is no uniform standard of clinical diagnosis and treatment of HIV/ AIDS with TCM; its application is still based on the treatment of other diseases. In view of this, we should collaborate with clinicians to formulate national unified efficacy standards for treating HIV/AIDS with TCM. We need to screen Chinese medicine compounds further, including single herbs and effective components of multiple agents, and look for treatment strategies that are suitable for China. At the same time, research on the standardization of anti-HIV medicine, and into determining the active components of Chinese medicine and their structure, should be advanced in order to maximize the potential benefits of TCM.

\section{REFERENCES}

[1] L. O. Kallings, "The First Postmodern Pandemic: 25 Years of HIV/AIDS,” Journal of Internal Medicine, Vol. 263, No. 3, 2008, pp. 218-243. doi:10.1111/j.1365-2796.2007.01910.x

[2] R. A. Weiss, "How Does HIV Cause AIDS?” Science, Vol. 260, No. 5112, 1993, pp. 1273-1279. doi:10.1126/science.8493571

[3] Ministry of Health of the People's Republic of China, "China Has Made Remarkable Achievements in AIDS Prevention and Treatment," 2010.

http://www.moh.gov.cn/publicfiles/business/htmlfiles/mo hbgt/s3582/201011/49893.html

[4] R. S. Hogg, K. V. Heath, B. Yip, et al., "Improved Survival among HIV-Infected Individuals Following Initiation of Antiretroviral Therapy," The Journal of the American Medical Association, Vol. 279, No. 6, 1998, pp. 450-454. doi:10.1001/jama.279.6.450

[5] A. Mocroft, S. Vella, T. L. Benfield, et al., "Changing Patterns of Mortality across Europe in Patients Infected with HIV-1. EuroSIDA Study Group,” Lancet, Vol. 352, No. 9142, 1998, pp. 1725-1730. doi:10.1016/S0140-6736(98)03201-2

[6] F. J. Palella Jr, K. M. Delaney, A. C. Moorman, et al., "Declining Morbidity and Mortality among Patients with Advanced Human Immunodeficiency Virus Infection. HIV Outpatient Study Investigators,” The New England Journal of Medicine, Vol. 338, No. 13, 1998, pp. 853-860. doi:10.1056/NEJM199803263381301

[7] R. Chou, L. H. Huffman, R. Fu, et al., "Screening for HIV: A Review of the Evidence for the U.S. Preventive Services Task Force," Annals of Internal Medicine, Vol. 143, No. 1, 2005, pp. 55-73.

[8] T. Hawkins, "Appearance-Related Side Effects of HIV-1 Treatment,” AIDS Patient Care and STDs, Vol. 20, No. 1, 2006, pp. 6-18. doi:10.1089/apc.2006.20.6

[9] A. d’Arminio Monforte, A. C. Lepri, G. Rezza, et al., "Insights into the Reasons for Discontinuation of the First Highly Active Antiretroviral Therapy (HAART) Regimen in a Cohort of Antiretroviral Naive Patients. I. CO. N. A. Study Group. Italian Cohort of Antiretroviral-Naive Patients,” Aids, Vol. 14, No. 5, 2000, pp. 499-507.

[10] I. W. Park, C. Han, X. Song, et al., "Inhibition of HIV-1 Entry by Extracts Derived from Traditional Chinese Medicinal Herbal Plants,” BMC Complementary and Alternative Medicine, Vol. 9, 2009, p. 29. doi:10.1186/1472-6882-9-29

[11] A. Vickers, "Recent Advances: Complementary Medicine,” British Medical Journal, Vol. 321, No. 7272, 2000, pp. 683-686. doi:10.1136/bmj.321.7262.683

[12] J. P. Liu, “The Use of Herbal Medicines in Early Drug Development for the Treatment of HIV Infections and AIDS,” Expert Opinion on Investigational Drugs, Vol. 16, No. 9, 2007, pp. 1355-1364. doi:10.1517/13543784.16.9.1355

[13] J. Wang, W. Zou and Y. Liu, "Use of Traditional Chinese Medicine in HIV/AIDS in China," Journal of Biomedical Science and Engineering, Vol. 3, No. 8, 2010, pp. 828831. doi:10.4236/jbise.2010.38111

[14] J. Wang, Y. Liu, W. Zou, et al., "Overview of AIDS treatment with Traditional Chinese medicine," Chinese Journal of AIDS \& STD, Vol. 16, No. 3, 2010, pp. 313315.

[15] S. D. Luo and P. JU, “Compared Traditional Chinese 
Medicine and Medicine to Treating AIDS," Journal of Henan University of Chinese Medicine, Vol. 21, No. 3, 2006, pp. 1-4.

[16] S. D. Luo, D. Q. Fan, H. Y. Wang, et al., "Research on Herbals of Anti-HIV Activity,” Yunnan Science and Technology Press, Kunming, 1998, p. 6.

[17] L. Gai, C. Q. Song, Z. B. Hu, et al., "Review of Anti-HIV Plants,” Foreign Medical Sciences, Vol. 23, No. 3, 2001, pp. 139-142.

[18] J. H. He and E. D. Mai, "Review of AIDS Treatment with Chinese medicine," Chinese Remedies \& Clinics, Vol. 4, No. 6, 2004, pp. 454-456.

[19] W. B. Lv, “Advances on Treating AIDS by TCM,” Journal of Traditional Chinese Medicine, Vol. 38, No. 6, 1997, pp. 368-371.

[20] L. Y. Kong, Z. Yang and Z. D. Min, "Thinking Of the Active Ingredients of TCM Modernization," Chinese Traditional and Herbal Drugs, Vol. 29, No. 5, 1998, pp. 354-355.

[21] W. B. Lv, “Advances in AIDS Treatment Abroad,” Foreign Medical Sciences, Vol. 19, No. 3, 1997, pp. 28-29.

[22] D. Shi and Z. L. Peng, "Randomized Double Blind Placebo Paralleled Clinical Research on HIV/AIDS with Qiankunning Tablets,” Study Journal of Traditional Chinese Medicine, Vol. 21, No. 9, 2003, pp. 1472-1474.

[23] M. Li, Z. Y. Wu, B. Liang, et al., "Treatment of AIDS with Fufangsanhuangsan (FSHS) - A Report of 41 Cases," Chinese Journal of AIDS\&STD, Vol. 12, No. 1, 2006, pp. 21-23.

[24] H. Wu, F. J. Zhang, C. Yao, et al., "Clinical Study of Tangcao Tablets in Treating HIV/AIDS,” In: Compilation of Theses of Treatment and Research on AIDS, Chinese Ancient Books Press, Beijing, 2004, pp. 95-103.

[25] J. Wang, Y. Liu, W. Zou, et al., "Clinical Observations on $100 \mathrm{HIV} / \mathrm{AIDS}$ Cases Treated with Chinese Herb Aining Granule plus HAART," Chinese Journal of AIDS \& STD, Vol. 14, No. 2, 2008, pp. 101-107.

[26] J. Wang, F. Z. Yang, M. Zhao, et al., "Randomized Double-Blinded and Controlled Clinical Trial on Treatment of HIV/AIDS by Zhongyan-4," Chinese Journal of Integrative Medicine, Vol. 12, No. 1, 2006, pp. 6-11. doi:10.1007/BF02857422

[27] J. Z. Chen, "Clinical Research of Xiaomi Granule on HIV/AIDS Oral Candidiasis,” Guangming Journal of Chinese Medicine, Vol. 24, No. 4, 2009, pp. 633-636.

[28] F. Jiang, S. H. Wei, B. Peng, et al., "Effect of Xiaomi Granules in Treating 40 Patients of HIV /AIDS Oral Candidiasis," Chinese Journal of Integrated Traditional and Western Medicine, Vol. 29, No. 12, 2009, pp. 11171119.

[29] Z. N. Chen, Y. Y. He and Y. R. Wei, "Chinese Medicine Gargle Treatment of 102 Cases AIDS with Oral Lesions,” Journal of Sichuan of Traditional Chinese Medicine, Vol. 28, No. 10, 2010, pp. 114-115.

[30] F. M. Wang, "Clinical Research of TCM on AIDS with Peripheral Neuropathy," Nei Mongol Journal of Tradi- tional Chinese Medicine, Vol. 27, No. 1, 2008, pp. 15-16.

[31] B. Qu and M. L. Zhang, "Integrative Medicine Treatment of 65 Cases AIDS with Pneumonia,” Traditional Chinese Medicinal Research, Vol. 21, No. 2, 2008, pp. 38-39.

[32] X. P. Yang, F. Jiang, C. J. Zhou, et al., "Modified Banxiaxiexin Tang Treatment of 49 Cases AIDS with Gastrointestinal Adverse Reactions Due to HAART," Traditional Chinese Medicinal Research, Vol. 21, No. 12, 2008, pp. 23-26.

[33] Z. Y. Fan, W. Ren and Y. L. Xing, "Integrative Medicine Treatment of 48 Cases HIV/AIDS with Diarrhea," Henan Traditional Chinese Medicine, Vol. 28, No. 5, 2008, pp. 55-56.

[34] G. H. Yang, M. Cui, L. H. Zhou, et al., "Efficacy on Shenlingbaishusan Treatment of AIDS-Related Diarrhea," Chinese Archives Of Traditional Chinese Medicine, Vol. 26, No. 1, 2008, pp. 150-153.

[35] T. S. Qiu and X. Z. Li, "Integrative Medicine Treatment of 52 Cases HIV/AIDS with Opportunistic Infections Diarrhea," Journal of Sichuan of Traditional Chinese Medicine, Vol. 28, No. 8, 2010, pp. 67-68.

[36] F. Z. Yang, J. Wang and Naomi, "Dynamic Observation on Tlymph Cell of HIV/AIDS Patients in Tanzania under Long-Term Chinese Medicine Intervention,” China Journal of Traditional Chinese Medicine and Pharmacy, Vol. 24, No. 12, 2009, pp. 1655-1658.

[37] S. J. Huang, J. A. Wei, H. Y. Cao, et al., "Clinical Research on TCM in the Treatment of HIV/AIDS," Journal of Traditional Chinese Medicine, Vol. 45, No. 9, 2004, pp. 680-682.

[38] S. P. Xie, "The Train of Thought in the Study on Treating AIDS with Traditional Chinese Medicine," Journal of Henan College of Traditional Chinese Medicine, Vol. 20, No. 2, 2005, pp. 1-3.

[39] Y. Guo and B. Y. Qian, “A Clinical Observation on 60 Cases of Treating Diarrhea Caused by AIDS with Moxibustion," Journal of Henan University of Chinese Medicine, Vol. 20, No. 4, 2005, pp. 6-7.

[40] J. D. Wang, G. E. Huang, J. Q. Zhai, et al., “Observation of the Curative Effect of Treating 50 Cases of Diarrhea of Acquired Immunodeficiency Syndrome with Moxa Roll Moxibustion," Journal of Henan University of Chinese Medicine, Vol. 21, No. 1, 2006, p. 14.

[41] L. H. Zhou, Y. Tang, G. H. Yang, et al., "Clinical Study of AIDS Associated Diarrhea Treated with TCM Therapy," Chinese Journal of Information on TCM, Vol. 14, No. 9, 2007, pp. 10-12.

[42] J. R. Wang, X. R. Chen, Q. Zhang, et al., "Effect of Moxibustion on Immunological Function in the Patient of AIDS of Spleen-Kidney Yang-Deficiency," Chinese Acupuncture \& Moxibustion, Vol. 27, No. 12, 2007, pp. 892894.

[43] J. Wang, B. Y. Liang, S. Y. Yan, et al., "Clinical Observation on 8946 AIDS Cases Treated by Traditional Chinese Medicine,” Journal of Traditional Chinese Medicine, Vol. 52, No. 5, 2011, pp. 395-398. 
[44] Y. W. Cen, L. C. Fu， X. H. Tan, et al., "Investigation of TCM Syndrome in HIV/AIDS Patients of Guangdong,"
Chinese Archives of Traditional Chinese Medicine, Vol. 26, No. 5, 2008, pp. 958-961. 